\title{
A Torres Condition for Twisted Alexander Polynomials
}

\author{
Dedicated to Professor Tomoyuki Wada on his 60th birthday
}

By

Takayuki MorIfuJI*

\begin{abstract}
As a generalization of a fundamental result about the Alexander polynomial of links, we give a description of a Torres condition for the twisted Alexander polynomial of links associated to a unimodular representation.
\end{abstract}

\section{$\S 1 . \quad$ Introduction}

The theory of twisted Alexander polynomial was introduced by Lin [13] and Wada [18]. Lin defined it for knots in the 3-sphere using regular Seifert surfaces. On the other hand, Wada defined the twisted Alexander polynomial for finitely presentable groups, which include the link groups. In particular, as an application, Wada told the Kinoshita-Terasaka knot from the Conway knot by means of his invariant. Shortly afterward, several significant results on the original Alexander polynomial were generalized to the twisted case. For example, equivalence of the twisted Alexander polynomial and the Reidemeister torsion, and its symmetry [9], [7], sliceness obstruction for knots and a relation to the Casson-Gordon invariant [7], [8], monicness of the twisted Alexander polynomial for fibered knots [1], [2] and so on. Recently the twisted Alexander polynomials are extensively investigated. See for instance [3], [4], [5], [6], [10], [11], [12], [14], [15] and [16].

Communicated by K. Saito. Received June 24, 2005. Revised October 17, 2005.

2000 Mathematics Subject Classification(s): Primary 57M25; Secondary 57M05, 57M27.

* Department of Mathematics, Tokyo University of Agriculture and Technology, Koganei,

Tokyo 184-8588, Japan.

e-mail: morifuji@cc.tuat.ac.jp

(c) 2007 Research Institute for Mathematical Sciences, Kyoto University. All rights reserved. 

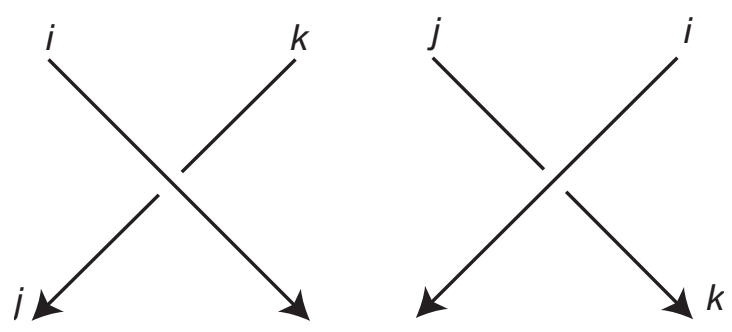

Figure 1.

However, almost all results mentioned above are basically about knots in the 3 -sphere and it seems that there are few generalized results on links. The purpose of the present paper is to give a generalization of the following wellknown formula for the Alexander polynomial of links.

Theorem 1.1 (Torres [17]). The Alexander polynomial $\Delta_{L}\left(t_{1}, \ldots, t_{\mu}\right)$ of a $\mu$-component link $L=L_{1} \cup \cdots \cup L_{\mu}$ satisfies

$$
\Delta_{L}\left(t_{1}, \ldots, t_{\mu-1}, 1\right)= \begin{cases}\frac{t_{1}^{l_{1}}-1}{t_{1}-1} \Delta_{L^{\prime}}\left(t_{1}\right) & \text { if } \mu=2 \\ \left(t_{1}^{l_{1}} \cdots t_{\mu-1}^{l_{\mu-1}}-1\right) \Delta_{L^{\prime}}\left(t_{1}, \ldots, t_{\mu-1}\right) & \text { if } \mu>2,\end{cases}
$$

where $L^{\prime}=L_{1} \cup \cdots \cup L_{\mu-1}$ is the link obtained from $L$ by removing $L_{\mu}$ and $l_{i}$ denotes the linking number of the components $L_{i}$ and $L_{\mu}$.

More precisely, we give a description of a Torres condition for the twisted Alexander polynomial of links associated to a unimodular representation. In the next section, we briefly recall the definition of the twisted Alexander polynomial for a link group. The precise statement and the proof of the main theorem of this paper are given in Section 3.

\section{$\S 2 . \quad$ Twisted Alexander Polynomial for Links}

Let $L=L_{1} \cup \cdots \cup L_{\mu}$ be a $\mu$-component link in the 3 -sphere. We denote the fundamental group of its exterior $E$ by $G(L)$. Namely, we put $G(L)=\pi_{1}(E)$ and call it the link group. We choose and fix a Wirtinger presentation of $G(L)$. That is, given a regular projection of the link $L$, we assign to each overpass a generator $x_{i}$ as in Figure 1 , a relator $x_{i} x_{k} x_{i}^{-1} x_{j}^{-1}$ or $x_{i}^{-1} x_{j} x_{i} x_{k}^{-1}$. Thus we obtain a presentation of $G(L)$ with $u$ generators and $u$ relators,

$$
\left\langle x_{1}, \ldots, x_{u} \mid r_{1}, \ldots, r_{u}\right\rangle .
$$


After some reordering of the indicies, the relators $r_{1}, \ldots, r_{u}$ satisfy

$$
\prod_{i=1}^{u} r_{i}^{ \pm 1}=1
$$

This means that any one of the relators is a consequence of the other $u-1$ relators. We remove one of the relators and call the resulting presentation

$$
G(L)=\left\langle x_{1}, \ldots, x_{u} \mid r_{1}, \ldots, r_{u-1}\right\rangle
$$

a Wirtinger presentation of $G(L)$.

The abelianization homomorphism

$$
\alpha: G(L) \rightarrow H_{1}(E ; \mathbb{Z}) \cong \mathbb{Z}^{\oplus \mu}=\left\langle t_{1}\right\rangle \oplus \cdots \oplus\left\langle t_{\mu}\right\rangle
$$

is given by assigning to each generator $x_{i}$ the meridian element $t_{k} \in H_{1}(E ; \mathbb{Z})$ of the corresponding component $L_{k}$ of $L$. In this paper, we consider a linear representation $\rho: G(L) \rightarrow S L(n ; F)$, where $F$ denotes a field.

These maps naturally induce two ring homomorphisms $\tilde{\rho}: \mathbb{Z}[G(L)] \rightarrow$ $M(n ; F)$ and $\tilde{\alpha}: \mathbb{Z}[G(L)] \rightarrow \mathbb{Z}\left[t_{1}^{ \pm 1}, \ldots, t_{\mu}^{ \pm 1}\right]$, where $\mathbb{Z}[G(L)]$ is the group ring of $G(L)$ over $\mathbb{Z}$ and $M(n ; F)$ is the matrix algebra of degree $n$ over $F$. Taking the tensor of $\tilde{\rho}$ and $\tilde{\alpha}$, we obtain a ring homomorphism

$$
\tilde{\rho} \otimes \tilde{\alpha}: \mathbb{Z}[G(L)] \rightarrow M\left(n ; F\left[t_{1}^{ \pm 1}, \ldots, t_{\mu}^{ \pm 1}\right]\right) .
$$

Let $F_{u}$ denote the free group on generators $x_{1}, \ldots, x_{u}$ and

$$
\Phi: \mathbb{Z}\left[F_{u}\right] \rightarrow M\left(n ; F\left[t_{1}^{ \pm 1}, \ldots, t_{\mu}^{ \pm 1}\right]\right)
$$

the composite of the surjection $\mathbb{Z}\left[F_{u}\right] \rightarrow \mathbb{Z}[G(L)]$ induced by the presentation and the map $\tilde{\rho} \otimes \tilde{\alpha}$.

Let us consider the $(u-1) \times u$ matrix $M=M\left(t_{1}, \ldots, t_{\mu}\right)$ whose $(i, j)$ th component is the $n \times n$ matrix

$$
\Phi\left(\frac{\partial r_{i}}{\partial x_{j}}\right) \in M\left(n ; F\left[t_{1}^{ \pm 1}, \ldots, t_{\mu}^{ \pm 1}\right]\right),
$$

where $\partial / \partial x$ denotes the free differential calculus. This matrix $M$ is called the Alexander matrix of $G(L)$ associated to the representation $\rho$.

For $1 \leq j \leq u$, let us denote by $M_{j}=M_{j}\left(t_{1}, \ldots, t_{\mu}\right)$ the $(u-1) \times(u-1)$ matrix obtained from $M$ by removing the column corresponding to a generator $x_{j}$. We also regard $M_{j}$ as an $n(u-1) \times n(u-1)$ matrix with coefficients in $F\left[t_{1}^{ \pm 1}, \ldots, t_{\mu}^{ \pm 1}\right]$. 
Then Wada's twisted Alexander polynomial of a link $L$ for a representation $\rho: G(L) \rightarrow S L(n ; F)$ is defined to be a rational function

$$
\Delta_{L, \rho}\left(t_{1}, \ldots, t_{\mu}\right)=\frac{\left|M_{j}\right|}{\left|\Phi\left(x_{j}-1\right)\right|},
$$

where $\left|M_{j}\right|$ denotes the determinant of the matrix $M_{j}$, and it is well-defined up to a factor $\pm t_{1}^{n k_{1}} \cdots t_{\mu}^{n k_{\mu}}\left(k_{i} \in \mathbb{Z}\right)$ if $n$ is odd and up to only $t_{1}^{n k_{1}} \cdots t_{\mu}^{n k_{\mu}}$ if $n$ is even (see [18] Section 5 for details).

Remark 2.1. In general, the twisted Alexander polynomial for a finitely presentable group is a rational function, but it is actually a polynomial for a link group (see [18] Proposition 9 and [10] Theorem 3.1).

\section{§3. A Torres Condition}

In this section, we state and prove a generalized Torres condition for the twisted Alexander polynomial of links. An advantage of our description here is that we need not separate the case for $\mu=2$ from the one for $\mu>2$. We first prove the theorem in the case of an $S L(2 ; F)$-representation. After reading the proof for it, one can easily show the similar result for general cases.

Theorem 3.1. Let $L=L_{1} \cup \cdots \cup L_{\mu}$ be a $\mu$-component link and $L^{\prime}=$ $L_{1} \cup \cdots \cup L_{\mu-1}$. For a given representation $\rho^{\prime}: G\left(L^{\prime}\right) \rightarrow S L(2 ; F)$, it holds that $\Delta_{L, \rho}\left(t_{1}, \ldots, t_{\mu-1}, 1\right)=\left\{\left(t_{1}^{l_{1}} \cdots t_{\mu-1}^{l_{\mu-1}}\right)^{2}+\varepsilon_{\rho^{\prime}} t_{1}^{l_{1}} \cdots t_{\mu-1}^{l_{\mu-1}}+1\right\} \Delta_{L^{\prime}, \rho^{\prime}}\left(t_{1}, \ldots, t_{\mu-1}\right)$, where $\rho: G(L) \rightarrow S L(2 ; F)$ is the composite of the natural surjection $G(L) \rightarrow$ $G\left(L^{\prime}\right)$ and $\rho^{\prime}, l_{i}$ denotes the linking number of $L_{i}$ and $L_{\mu}$, and $\varepsilon_{\rho^{\prime}}$ is an element of $F$.

Proof. For the link group $G(L)$, we choose a Wirtinger presentation:

$$
G(L)=\left\langle x_{i j} \mid r_{k l}\right\rangle
$$

where $x_{i 1}, x_{i 2}, \ldots, x_{i j_{i}}(1 \leq i \leq \mu)$ are generators corresponding to the component $L_{i}$ and the relator

$$
r_{k l}=x_{k^{\prime} l^{\prime}} x_{k l} x_{k^{\prime} l^{\prime}}^{-1} x_{k, l+1}^{-1} \quad \text { or } \quad x_{k^{\prime} l^{\prime}}^{-1} x_{k l} x_{k^{\prime} l^{\prime}} x_{k, l+1}^{-1}
$$

corresponds to a crossing of $L_{k^{\prime}}$ over $L_{k}$. In the above presentation, we arrange the generators and relators in lexicographic order, which is determined by the 
order of components $L_{1}, \ldots, L_{\mu}$ and the orientation of each component $L_{i}$. We should note that the link group $G(L)$ has the deficiency one (namely, the number of relators is less than that of generators).

Let us consider the Alexander matrix of $G(L)$ associated to the representation $\rho: G(L) \rightarrow S L(2 ; F)$ :

$$
\begin{aligned}
M\left(t_{1}, \ldots, t_{\mu}\right)= & \left(\Phi\left(\frac{\partial r_{k l}}{\partial x_{i j}}\right)\right) \\
& =\left(\begin{array}{cc}
\Phi\left(\frac{\partial r_{k l}}{\partial x_{i j}}\right)_{k, i \neq \mu} & \Phi\left(\frac{\partial r_{k l}}{\partial x_{\mu j}}\right)_{k \neq \mu, 1 \leq j \leq j_{\mu}} \\
\Phi\left(\frac{\partial r_{\mu l}}{\partial x_{i j}}\right)_{i \neq \mu, 1 \leq l \leq j_{\mu}} & \Phi\left(\frac{\partial r_{\mu l}}{\partial x_{\mu j}}\right)_{1 \leq j, l \leq j_{\mu}}
\end{array}\right) .
\end{aligned}
$$

Then we know that if we remove the column corresponding to a generator $x_{i j}(i \neq \mu)$,

$$
\left|M_{i j}\left(t_{1}, \ldots, t_{\mu}\right)\right|=\left|\Phi\left(x_{i j}-1\right)\right| \Delta_{L, \rho}\left(t_{1}, \ldots, t_{\mu}\right)
$$

holds. Thus setting $t_{\mu}=1$ in $M\left(t_{1}, \ldots, t_{\mu}\right)$, it follows that

$$
\left|M_{i j}\left(t_{1}, \ldots, t_{\mu-1}, 1\right)\right|=\left|\Phi\left(x_{i j}-1\right)\right| \Delta_{L, \rho}\left(t_{1}, \ldots, t_{\mu-1}, 1\right)
$$

if $i \neq \mu$.

Now the generators $\left\{x_{\mu j}\right\}\left(1 \leq j \leq j_{\mu}\right)$ appear in the following two kinds of relators:

$$
\text { (i) } r_{\mu j}=x_{v w}^{ \pm 1} x_{\mu j} x_{v w}^{\mp 1} x_{\mu, j+1}^{-1} \quad \text { and } \quad\left(\text { ii) } r_{p q}=x_{\mu l}^{ \pm 1} x_{p q} x_{\mu l}^{\mp 1} x_{p, q+1}^{-1}\right. \text {, }
$$

where the relator (i) corresponds to crossings of $L_{v}$ over $L_{\mu}$ and (ii) corresponds to that of $L_{\mu}$ over $L_{p}$. Let us see which are the contributions of these relators to the matrix $M\left(t_{1}, \ldots, t_{\mu-1}, 1\right)$.

Claim 1. The contributions of $r_{\mu j}$ are as follows:

(i) $\Phi\left(\frac{\partial r_{\mu j}}{\partial x_{v w}}\right)_{t_{\mu}=1}=O$,

(ii) $\Phi\left(\frac{\partial r_{\mu j}}{\partial x_{\mu j}}\right)_{t_{\mu}=1}= \begin{cases}t_{v}^{ \pm 1} \rho\left(x_{v w}\right)^{ \pm 1} & \text { if } \mu \neq v \\ I & \text { if } \mu=v\end{cases}$

(iii) $\Phi\left(\frac{\partial r_{\mu j}}{\partial x_{\mu, j+1}}\right)_{t_{\mu}=1}=-I$,

where $O$ and $I$ denote the zero and the identity matrix respectively. 
Proof. (i) An easy calculation shows that

$$
\frac{\partial r_{\mu j}}{\partial x_{v w}}=1-x_{v w} x_{\mu j} x_{v w}^{-1} \quad \text { or } \quad-x_{v w}^{-1}+x_{v w}^{-1} x_{\mu j} .
$$

Putting $t_{\mu}=1$, we obtain

$$
\Phi\left(\frac{\partial r_{\mu j}}{\partial x_{v w}}\right)_{t_{\mu}=1}=I-t_{v} t_{v}^{-1} \rho\left(x_{v w}\right) \rho\left(x_{\mu j}\right) \rho\left(x_{v w}\right)^{-1}=O
$$

or

$$
\Phi\left(\frac{\partial r_{\mu j}}{\partial x_{v w}}\right)_{t_{\mu}=1}=-t_{v}^{-1} \rho\left(x_{v w}\right)^{-1}+t_{v}^{-1} \rho\left(x_{v w}\right)^{-1} \rho\left(x_{\mu j}\right)=O
$$

because $\rho\left(x_{\mu j}\right)=I$ for $1 \leq j \leq j_{\mu}$. (ii) and (iii) follow from the similar calculation. This completes the proof of Claim 1.

Claim 2. The contributions of $r_{p q}$ are as follows:

(i) $\Phi\left(\frac{\partial r_{p q}}{\partial x_{\mu l}}\right)_{t_{\mu}=1}= \pm\left(I-t_{p} \rho\left(x_{p q}\right)\right)$,

(ii) $\Phi\left(\frac{\partial r_{p q}}{\partial x_{p q}}\right)_{t_{\mu}=1}=I$,

(iii) $\Phi\left(\frac{\partial r_{p q}}{\partial x_{p, q+1}}\right)_{t_{\mu}=1}=-\rho\left(x_{p q}\right) \rho\left(x_{p, q+1}\right)^{-1}$ if $p \neq \mu$

and the case $p=\mu$ has already been considered.

Proof. We only show (iii). Since

$$
\frac{\partial r_{p q}}{\partial x_{p, q+1}}=-x_{\mu l}^{ \pm 1} x_{p q} x_{\mu l}^{\mp 1} x_{p, q+1}^{-1},
$$

putting $t_{\mu}=1$ and using $\rho\left(x_{\mu l}\right)=I$, we have

$$
\begin{aligned}
\Phi\left(\frac{\partial r_{p q}}{\partial x_{p, q+1}}\right)_{t_{\mu}=1} & =-t_{p} t_{p}^{-1} \rho\left(x_{\mu l}\right)^{ \pm 1} \rho\left(x_{p q}\right) \rho\left(x_{\mu l}\right)^{\mp 1} \rho\left(x_{p, q+1}\right)^{-1} \\
& =-\rho\left(x_{p q}\right) \rho\left(x_{p, q+1}\right)^{-1}
\end{aligned}
$$

if $p \neq \mu$. The proof of Claim 2 is completed.

From the above two claims, we see that the matrix $M\left(t_{1}, \ldots, t_{\mu-1}, 1\right)$ has the following form:

$$
M\left(t_{1}, \ldots, t_{\mu-1}, 1\right)=\left(\begin{array}{ll}
A & B \\
O & C
\end{array}\right)
$$


where

$A=\left(\Phi\left(\frac{\partial r_{k l}}{\partial x_{i j}}\right)_{t_{\mu}=1}\right)(k, i \neq \mu), B=\left(\Phi\left(\frac{\partial r_{k l}}{\partial x_{\mu j}}\right)_{t_{\mu}=1}\right)\left(k \neq \mu, 1 \leq j \leq j_{\mu}\right)$,

and

$$
\begin{aligned}
& C=\left(\Phi\left(\frac{\partial r_{\mu l}}{\partial x_{\mu j}}\right)_{t_{\mu}=1}\right)\left(1 \leq j, l \leq j_{\mu}\right)
\end{aligned}
$$

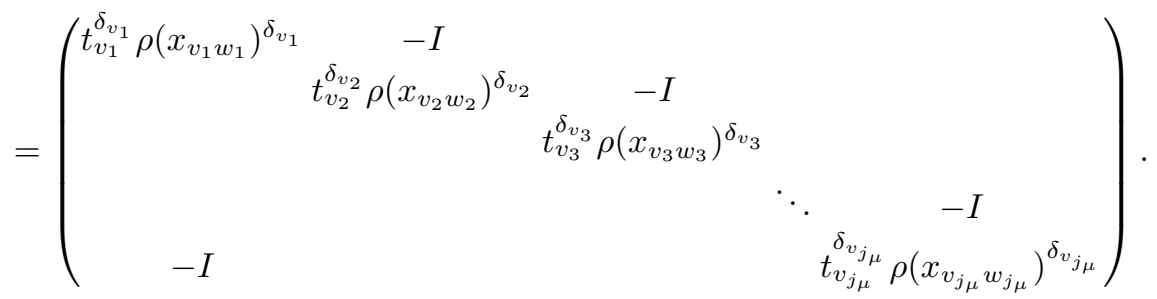

Here $\delta_{v_{i}}=1$ or -1 according to crossings of $L_{v_{i}}$ over $L_{\mu}$.

Claim 3. The determinant of the submatrix $C$ is given by

$$
|C|=\left(t_{1}^{l_{1}} \cdots t_{\mu-1}^{l_{\mu-1}}\right)^{2}+\varepsilon_{\rho^{\prime}} t_{1}^{l_{1}} \cdots t_{\mu-1}^{l_{\mu-1}}+1
$$

where $\varepsilon_{\rho^{\prime}}$ is an element of $F$.

Proof. By definition of the determinant of a matrix, we have

$$
\begin{aligned}
|C|= & \prod_{i=1}^{j_{\mu}}\left|\rho\left(x_{v_{i} w_{i}}\right)^{\delta_{v_{i}}}\right| t_{v_{i}}^{2 \delta_{v_{i}}}+(-1)^{j_{\mu}} \sum_{\sigma \in S}(\operatorname{sgn} \sigma) \gamma_{1}^{\sigma} \cdots \gamma_{j_{\mu}}^{\sigma} t_{v_{1}}^{\delta_{v_{1}}} \cdots t_{v_{j_{\mu}}}^{\delta_{v_{j_{\mu}}}} \\
& +\left(\operatorname{sgn} \sigma_{0}\right)(-1)^{2 j_{\mu}}
\end{aligned}
$$

where $\gamma_{i}^{\sigma} \in F$ denotes a component of the $2 \times 2$-matrix $\rho\left(x_{v_{i} w_{i}}\right)^{\delta_{v_{i}}}$ determined by a permutation $\sigma, S$ is a subset of the symmetric group $\mathfrak{S}_{2 j_{\mu}}$ consisting of permutations which choose just one component from each submatrix $\rho\left(x_{v_{i} w_{i}}\right)^{\delta_{v_{i}}}$ and

$$
\sigma_{0}=\left(135 \ldots 2 j_{\mu}-1\right)\left(246 \ldots 2 j_{\mu}\right) \in \mathfrak{S}_{2 j_{\mu}} .
$$

For example, when $j_{\mu}=2$, the permutation $\sigma=(1342) \in S \subset \mathfrak{S}_{4}$ assigns the coefficient

$$
\gamma_{1}^{\sigma} \gamma_{2}^{\sigma}=c_{1} b_{2},
$$

where $c_{1}, b_{2}$ are components of the images

$$
\rho\left(x_{v_{i} w_{i}}\right)^{\delta_{v_{i}}}=\left(\begin{array}{ll}
a_{i} & b_{i} \\
c_{i} & d_{i}
\end{array}\right), \quad(i=1,2) .
$$


On the other hand, in the matrix $C$, there is an appearance of $t_{i}^{\delta_{i}}$ for each crossing of $L_{i}$ over $L_{\mu}(1 \leq i \leq \mu)$ and $\delta_{i}=1$ or -1 according as $L_{i}$ crosses over $L_{\mu}$ from left to right or from right to left. Thus $t_{v_{1}}^{\delta_{v_{1}}} \cdots t_{v_{j_{\mu}}}^{\delta_{j_{\mu}}}=t_{1}^{l_{1}} \cdots t_{\mu-1}^{l_{\mu-1}}$ holds. Since sgn $\sigma_{0}=1$, if we put

$$
\varepsilon_{\rho^{\prime}}=(-1)^{j_{\mu}} \sum_{\sigma \in S}(\operatorname{sgn} \sigma) \gamma_{1}^{\sigma} \cdots \gamma_{j_{\mu}}^{\sigma} \in F
$$

we obtain

$$
|C|=\left(t_{1}^{l_{1}} \cdots t_{\mu-1}^{l_{\mu-1}}\right)^{2}+\varepsilon_{\rho^{\prime}} t_{1}^{l_{1}} \cdots t_{\mu-1}^{l_{\mu-1}}+1 .
$$

This completes the proof of Claim 3 .

Next the matrix $A$ is equivalent to the Alexander matrix $M^{\prime}\left(t_{1}, \ldots, t_{\mu-1}\right)$ of $G\left(L^{\prime}\right)$ associated to the representation $\rho^{\prime}: G\left(L^{\prime}\right) \rightarrow S L(2 ; F)$. Hence if we remove a column corresponding to a generator $x_{i j}(i \neq \mu)$, then we have

$$
\begin{aligned}
\left|M_{i j}\left(t_{1}, \ldots, t_{\mu-1}, 1\right)\right| & =\left|A_{i j}\right||C| \\
& =\left\{\left(t_{1}^{l_{1}} \cdots t_{\mu-1}^{l_{\mu-1}}\right)^{2}+\varepsilon_{\rho^{\prime}} t_{1}^{l_{1}} \cdots t_{\mu-1}^{l_{\mu-1}}+1\right\}\left|M_{i j}^{\prime}\left(t_{1}, \ldots, t_{\mu-1}\right)\right|,
\end{aligned}
$$

where $A_{i j}$ is the matrix obtained from $A$ by removing the column corresponding to $x_{i j}$. Therefore, by definition of the twisted Alexander polynomial, we see that

$\Delta_{L, \rho}\left(t_{1}, \ldots, t_{\mu-1}, 1\right)=\left\{\left(t_{1}^{l_{1}} \cdots t_{\mu-1}^{l_{\mu-1}}\right)^{2}+\varepsilon_{\rho^{\prime}} t_{1}^{l_{1}} \cdots t_{\mu-1}^{l_{\mu-1}}+1\right\} \Delta_{L^{\prime}, \rho^{\prime}}\left(t_{1}, \ldots, t_{\mu-1}\right)$.

This completes the proof of Theorem 3.1.

Remark 3.2. The fact that $\Delta_{L, \rho}\left(t_{1}, \ldots, t_{\mu-1}, 1\right)$ is divisible by $\Delta_{L^{\prime}, \rho^{\prime}}\left(t_{1}\right.$, $\left.\ldots, t_{\mu-1}\right)$ also follows from a recent result of Kitano, Suzuki and Wada in [12]. However, we can have no detailed information on the quotient from their result.

A linear representation $\rho: G(L) \rightarrow G L(n ; F)$ is called reducible if it has a nontrivial invariant subspace in $F^{n}$. In this case, we can obtain a piece of information about the coefficient $\varepsilon_{\rho^{\prime}}$.

Corollary 3.3. Under the setting as in Theorem 3.1, if $\rho^{\prime}: G\left(L^{\prime}\right) \rightarrow$ $S L(2 ; F)$ is a reducible representation, then we have

$$
\varepsilon_{\rho^{\prime}}=-\left(\prod_{i=1}^{\mu-1} \lambda_{i}^{l_{i}}+\prod_{i=1}^{\mu-1} \lambda_{i}^{-l_{i}}\right),
$$

where $\lambda_{i}$ is an eigenvalue of the image of a generator $x_{i j}(i \neq \mu)$ of $G\left(L^{\prime}\right)$. 
Proof. First we can assume that the images of generators in a Wirtinger presentation of $G(L)$ have the following forms:

$$
\rho\left(x_{i j}\right)=\left(\begin{array}{cc}
a_{i j} & b_{i j} \\
0 & a_{i j}^{-1}
\end{array}\right) \quad(i \neq \mu) \quad \text { and } \quad \rho\left(x_{\mu j}\right)=I,
$$

where $a_{i j} \in F^{\times}$and $b_{i j} \in F$. Because the representation $\rho^{\prime}$ has a 1-dimensional invariant subspace in $F^{2}$.

Since $x_{i j} x_{i k}^{-1}(i \neq \mu, j \neq k)$ is an element of the commutator subgroup of $G(L),[G(L), G(L)]$, we see that $a_{i j}=a_{i k}$ holds for these generators. We then put $\lambda_{i}=a_{i j}$ for simplicity. Each lower left component of $\rho\left(x_{i j}\right)$ is zero, so that the nontrivial terms appeared in the coefficient of $t_{v_{1}}^{\delta_{v_{1}}} \cdots t_{v_{j_{\mu}}}^{\delta_{v_{j}}}$ are just

$$
(-1)^{j_{\mu}}\left(\operatorname{sgn} \sigma_{1}\right) \lambda_{v_{1}}^{\delta_{v_{1}}} \cdots \lambda_{v_{j_{\mu}}}^{\delta_{v_{j}}}+(-1)^{j_{\mu}}\left(\operatorname{sgn} \sigma_{2}\right) \lambda_{v_{1}}^{-\delta_{v_{1}}} \cdots \lambda_{v_{j_{\mu}}}^{-\delta_{v_{j_{\mu}}}},
$$

where $\sigma_{1}=\left(246 \ldots 2 j_{\mu}\right)$ and $\sigma_{2}=\left(135 \ldots 2 j_{\mu}-1\right)$ are elements of the symmetric group $\mathfrak{S}_{2 j_{\mu}}$. Then it is easy to check that $(-1)^{j_{\mu}} \operatorname{sgn} \sigma_{1}=(-1)^{j_{\mu}} \operatorname{sgn} \sigma_{2}=$ -1 holds. Therefore we can have the desired formula. This completes the proof.

Example 3.4. Let $\rho^{\prime}: G\left(L^{\prime}\right) \rightarrow S L(2 ; F)$ be a reducible representation of a knot $L^{\prime}=L_{1}$. Then the twisted Alexander polynomial of $L^{\prime}$ associated to $\rho^{\prime}$ is given by

$$
\Delta_{L^{\prime}, \rho^{\prime}}\left(t_{1}\right)=\frac{\Delta_{L^{\prime}}\left(\lambda t_{1}\right) \Delta_{L^{\prime}}\left(\lambda^{-1} t_{1}\right)}{\left(t_{1}-\lambda\right)\left(t_{1}-\lambda^{-1}\right)}
$$

where $\Delta_{L^{\prime}}\left(t_{1}\right)$ is the original Alexander polynomial of $L^{\prime}$ and $\lambda$ is an eigenvalue of the image of a generator of $G\left(L^{\prime}\right)$ (see the proof of [10] Theorem 3.1 for instance). Hence we have

$$
\begin{aligned}
\Delta_{L, \rho}(1,1) & =\left\{2-\left(\lambda^{l_{1}}+\lambda^{-l_{1}}\right)\right\} \Delta_{L^{\prime}, \rho^{\prime}}(1) \\
& =\frac{\left(1-\lambda^{l_{1}}\right)\left(1-\lambda^{-l_{1}}\right)}{(1-\lambda)\left(1-\lambda^{-1}\right)} \Delta_{L^{\prime}}(\lambda) \Delta_{L^{\prime}}\left(\lambda^{-1}\right) \\
& =\left(1+\lambda+\cdots+\lambda^{l_{1}-1}\right)\left(1+\lambda^{-1}+\cdots+\lambda^{-\left(l_{1}-1\right)}\right) \Delta_{L^{\prime}}(\lambda) \Delta_{L^{\prime}}\left(\lambda^{-1}\right) .
\end{aligned}
$$

In particular, if $\rho^{\prime}$ has the eigenvalue $\lambda=1$, then we obtain $\Delta_{L, \rho}(1,1)=l_{1}{ }^{2}$ (because $\Delta_{L^{\prime}}(1)= \pm 1$ ).

Example 3.5. Let $\rho^{\prime}: G\left(L^{\prime}\right) \rightarrow S L(2 ; F)$ be the trivial representation. In this case $\varepsilon_{\rho^{\prime}}=-2$ holds, so that we have

$$
\Delta_{L, \rho}\left(t_{1}, \ldots, t_{\mu-1}, 1\right)=\left(t_{1}^{l_{1}} \cdots t_{\mu-1}^{l_{\mu-1}}-1\right)^{2} \Delta_{L^{\prime}, \rho^{\prime}}\left(t_{1}, \ldots, t_{\mu-1}\right) .
$$


This formula corresponds to the square of Torres' original formula in Theorem 1.1. In particular, $\Delta_{L, \rho}(1, \ldots, 1)=0$ holds for $\mu>2$.

If we slightly modify the proof of Theorem 3.1, we obtain the following general formula for a unimodular representation $\rho^{\prime}: G\left(L^{\prime}\right) \rightarrow S L(n ; F)$. We omit here the repetitious proof.

Theorem 3.6. Let $L=L_{1} \cup \cdots \cup L_{\mu}$ be a $\mu$-component link and $L^{\prime}=$ $L_{1} \cup \cdots \cup L_{\mu-1}$. For a given representation $\rho^{\prime}: G\left(L^{\prime}\right) \rightarrow S L(n ; F)$, it holds that

$$
\begin{aligned}
\Delta_{L, \rho}\left(t_{1}, \ldots, t_{\mu-1}, 1\right)= & \left\{\left(t_{1}^{l_{1}} \cdots t_{\mu-1}^{l_{\mu-1}}\right)^{n}+\sum_{k=1}^{n-1} \varepsilon_{k, \rho^{\prime}}\left(t_{1}^{l_{1}} \cdots t_{\mu-1}^{l_{\mu-1}}\right)^{n-k}+(-1)^{n}\right\} \\
& \times \Delta_{L^{\prime}, \rho^{\prime}}\left(t_{1}, \ldots, t_{\mu-1}\right),
\end{aligned}
$$

where $\rho: G(L) \rightarrow S L(n ; F)$ is the composite of the natural surjection $G(L) \rightarrow$ $G\left(L^{\prime}\right)$ and $\rho^{\prime}, l_{i}$ denotes the linking number of $L_{i}$ and $L_{\mu}$, and $\varepsilon_{k, \rho^{\prime}}(1 \leq k \leq$ $n-1)$ are elements of $F$.

Remark 3.7. If $\rho$ is a representation to the general linear group $G L(n ; F)$, then the coefficient of the leading term $\left(t_{1}^{l_{1}} \cdots t_{\mu-1}^{l_{\mu-1}}\right)^{n}$ becomes a unit element $\varepsilon_{0, \rho^{\prime}} \in F^{\times}$.

Finally, we extend Corollary 3.3 when all the images of the representation $\rho^{\prime}: G\left(L^{\prime}\right) \rightarrow S L(n ; F)$ are upper triangle matrices.

Corollary 3.8. Under the setting as in Theorem 3.6, if $\operatorname{Im}\left(\rho^{\prime}\right)$ are upper triangle matrices, then the coefficient $\varepsilon_{k, \rho^{\prime}}$ is given by

$$
\varepsilon_{k, \rho^{\prime}}=(-1)^{k} \sum_{1 \leq j_{1}<\cdots<j_{k} \leq n} \prod_{i=1}^{\mu-1}\left(\lambda_{i 1} \cdots \hat{\lambda}_{i j_{1}} \cdots \hat{\lambda}_{i j_{k}} \cdots \lambda_{i n}\right)^{l_{i}},
$$

where $\lambda_{i m}(1 \leq m \leq n)$ are the eigenvalues of the image of a generator $x_{i j}(i \neq$ $\mu)$ of $G\left(L^{\prime}\right)$ and $\hat{\lambda}_{i m}$ implies that $\lambda_{i m}$ is removed from the product.

\section{Acknowledgements}

A part of this paper was written while the author was visiting the LudwigMaximilians-Universität in München. He would like to express his sincere thanks for their hospitality. The author also would like to thank the referee for useful comments. This research is partially supported by the Grant-in-Aid for Scientific Research (No. 17740032), the Ministry of Education, Culture, Sports, Science and Technology, Japan. 


\section{References}

[1] J. C. Cha, Fibred knots and twisted Alexander invariants, Trans. Amer. Math. Soc. 355 (2003), no. 10, 4187-4200 (electronic).

[2] H. Goda, T. Kitano and T. Morifuji, Reidemeister torsion, twisted Alexander polynomial and fibered knots, Comment. Math. Helv. 80 (2005), no. 1, 51-61.

[3] H. Goda and T. Morifuji, Twisted Alexander polynomial for SL(2, $\mathbb{C}$ )-representations and fibered knots, C. R. Math. Acad. Sci. Soc. R. Can. 25 (2003), no. 4, 97-101.

[4] M. Heusener and J. Porti, Deformations of reducible representations of 3-manifold groups into $\mathrm{PSL}_{2}(\mathbb{C})$, Algebr. Geom. Topol. 5 (2005), 965-997 (electronic).

[5] J. A. Hillman, C. Livingston and S. Naik, Twisted Alexander polynomials of periodic knots, Algebr. Geom. Topol. 6 (2006), 145-169 (electronic).

[6] B. J. Jiang and S. C. Wang, Twisted topological invariants associated with representations, in Topics in knot theory (Erzurum, 1992), 211-227, Kluwer Acad. Publ., Dordrecht.

[7] P. Kirk and C. Livingston, Twisted Alexander invariants, Reidemeister torsion, and Casson-Gordon invariants, Topology 38 (1999), no. 3, 635-661.

[8] - Twisted knot polynomials: inversion, mutation and concordance, Topology $\mathbf{3 8}$ (1999), no. 3, 663-671.

[9] T. Kitano, Twisted Alexander polynomial and Reidemeister torsion, Pacific J. Math. 174 (1996), no. 2, 431-442.

[10] T. Kitano and T. Morifuji, Divisibility of twisted Alexander polynomials and fibered knots, Ann. Sc. Norm. Super. Pisa Cl. Sci. (5) 4 (2005), no. 1, 179-186.

[11] T. Kitano and M. Suzuki, A partial order in the knot table, Experiment. Math. 14 (2005), no. 4, 385-390.

[12] T. Kitano, M. Suzuki and M. Wada, Twisted Alexander polynomials and surjectivity of a group homomorphism, Algebr. Geom. Topol. 5 (2005), 1315-1324 (electronic).

[13] X. S. Lin, Representations of knot groups and twisted Alexander polynomials, Acta Math. Sin. (Engl. Ser.) 17 (2001), no. 3, 361-380.

[14] T. Morifuji, A twisted invariant for finitely presentable groups, Proc. Japan Acad. Ser. A Math. Sci. 76 (2000), no. 9, 143-145.

[15] - Twisted Alexander polynomial for the braid group, Bull. Austral. Math. Soc. 64 (2001), no. 1, 1-13.

[16] M. Suzuki, Twisted Alexander polynomial for the Lawrence-Krammer representation, Bull. Austral. Math. Soc. 70 (2004), no. 1, 67-71.

[17] G. Torres, On the Alexander polynomial, Ann. of Math. (2) 57 (1953), 57-89.

[18] M. Wada, Twisted Alexander polynomial for finitely presentable groups, Topology 33 (1994), no. 2, 241-256. 